\title{
2-Methyl-3-Hydroxybutyryl-CoA Dehydrogenase (MHBD) Deficiency: An X-linked Inborn Error of Isoleucine Metabolism that May Mimic a Mitochondrial Disease
}

\author{
JUDIT GARCÍA-VILLORIA, ROB OFMAN, PEDRO RUIZ SALA, BEGOÑA MERINERO, JULIO RAMOS, \\ MARIA TERESA GARCÍA-SILVA, BEATRIZ BESELER, JAIME DALMAU, RONALD J.A. WANDERS, \\ AND MAGDALENA UGARTE \\ Institut de Bioquímica Clínica [J.G.-V.], Corporaciò Sanitària Clínic, Edifici Helios III, 08028 Barcelona, \\ Spain, University Hospital Amsterdam AMC [R.O., R.J.A.W.], Department of Pediatric and Clinical \\ Chemistry, 1105 AZ Amsterdam, The Netherlands, Centro de Diagnóstico de Enfermedades Moleculares \\ [C.P.-C., P.R.S., B.M., M.U.], Universidad Autónoma de Madrid, 28049 Madrid, Spain, Servicio de \\ Pediatría [J.R.], Hospital de Torrecárdenas, 04009 Almeria, Spain, Unidad de Enfermedades \\ Mitocondriales [M.T.G.-S.], Hospital 12 de Octubre, 28041 Madrid, Spain, Servicio de Pediatría [B.B], \\ Hospital de Denia [J.D.], 03700 Denia, Spain, Servicio de Pediatría, Hospital Infantil La Fe, 46009 \\ Valencia, Spain
}

\begin{abstract}
WBST
We describe three patients, from two Spanish families, with
2-methyl-3-hydroxybutyryl-CoA dehydrogenase (MHBD) defi-
ciency, a recently described $\mathrm{X}$-linked neurodegenerative inborn
error of isoleucine metabolism. Two of them are males with
severe lactic acidosis suggestive of a mitochondrial encephalop-
athy, and the third is a female who was less severely affected,
suggesting skewed $\mathrm{X}$-inactivation. Molecular studies revealed a
new missense mutation, $740 \mathrm{~A} \rightarrow \mathrm{G}$, in one family and a previ-
ously described mutation, $388 \mathrm{C} \rightarrow \mathrm{T}$, in the other, causing the
amino acid substitutions $\mathrm{N} 247 \mathrm{~S}$ and $\mathrm{R} 130 \mathrm{C}$, respectively. Both
\end{abstract}
\section{ABSTRACT}

male patients died, one of them despite treatment with an isoleucine-restricted diet, but the disease has remained stable in the female patient after 1 y of treatment. (Pediatr Res 58: 488-491, 2005)
Abbreviations
MHBD, 2-methyl-3-hydroxybutyryl-CoA dehydrogenase
2M3HBA, 2-methyl-3-hydroxybutyric acid
TG, tiglylglycine

MHBD (EC 1.1.1.178) deficiency is a recently described $\mathrm{X}$-linked inborn error in the metabolism of isoleucine (MIM 300256). MHBD is a mitochondrial enzyme that catalyzes the conversion of 2-methyl-3-hydroxybutyryl-CoA to 2-methylacetoacetyl-CoA. This disorder is characterized by normal early development followed by progressive loss of mental and motor skills. To our knowledge, only seven patients (1-6) including one female patient (3) have been reported. As expected in an $\mathrm{X}$-linked disease, males show lower enzyme activity and a more severe clinical course. However, a 23-y-old man with a milder phenotype has also been described (4).

Received August 9, 2004; accepted January 4, 2005

Correspondence: Antonia Ribes, Ph.D., Institut de Bioquímica Clínica, Corporaciò Sanitària Clínic, Edifici Helios III, planta baixa, C/ Mejía Lequerica s/n, 08028 Barcelona, Spain; e-mail: aribes@clinic.ub.es

Supported, in part, by FIS grant RED G-054B-O, REDEMETH, and an institutional grant from the Fundación Ramón Areces to the Centro de Biología Molecular.

DOI: 10.1203/01.pdr.0000176916.94328.cd
In addition to its role in isoleucine metabolism, MHBD seems to play a role in the pathogenesis of Alzheimer's disease (7) and it seems that the interaction of this protein with amyloid- $\beta$ peptide-binding protein may induce mitochondrial dysfunction (8). The gene encoding MHBD, is named $\mathrm{HADH} 2$, and has been mapped to chromosome Xp11.2. It spans about $3.11 \mathrm{~Kb}$ and consists of six exons. Only two different mutations have been reported to date (9).

An isoleucine-restricted diet has been administered to some patients $(1,3,4,6)$, which seems to stabilize the clinical progression of the disease.

Here we report three MHBD deficient patients, which adds new clinical, biochemical, and molecular data to expand our knowledge of this severe disease.

\section{PATIENTS AND METHODS}

Patient 1. Patient 1 is a female, born to nonconsanguineous healthy parents. Family history revealed a brother (patient 2) who died during the neonatal 
period due to lactic acidosis. The patient presented with psychomotor delay from the first months of life, walked at around $22 \mathrm{mo}$ and, at $2 \mathrm{y}$ and $5 \mathrm{mo}$, her gait was ataxic. Later on, speech delay and neurosensorial deafness were evident. During stress, she showed episodes of myoclonus. Brain MRI, performed at $2 \mathrm{y}$ and 5 mo of age, was normal.

Biochemical studies revealed slight hyperlactatemia: 1.7-3.0 mM (control range, 1-2 $\mathrm{mM}$ ) and microcytic anemia. Amino acids, carnitine, glucose, ammonia, creatin phospho kinase $(\mathrm{CPK})$, and other routine biochemical parameters were normal. Urinary organic acid profile showed an increase of 2M3HBA, $99 \mathrm{mmol} / \mathrm{mol}$ creatinine (control range, 5-12), and TG, $21 \mathrm{mmol} /$ mol creatinine (controls, $<5$ ). Lactate, fumarate, and 3-methylglutaconate were also increased. This profile initially suggested a complex I deficiency (10). Measurement of the respiratory chain activities in a muscle biopsy were normal (Unpublished results, performed by Dr. Ruitenbeek, Clinical Genetics Center, Nijmegen). At $9 \mathrm{y}$ of age, an oral isoleucine loading test was performed (1); plasma isoleucine rose to $980 \mu \mathrm{M}$ within the first $2 \mathrm{~h}$ (values for two independent controls: 757,812$)$ and was still high $(911 \mu \mathrm{M})$ after $4 \mathrm{~h}$ (values for two independent controls: 286, 375). Urinary excretion of $2 \mathrm{M} 3 \mathrm{HBA}$ and TG rose to 1285 and $74 \mathrm{mmol} / \mathrm{mol}$ creatinine, respectively, $6 \mathrm{~h}$ after isoleucine load (values for two independent controls: 53, 33 and 24, 25 respectively) (Fig 1). These results pointed to MHBD deficiency and a low-protein, isoleucine $(50 \mathrm{mg} / \mathrm{kg} /$ day) restricted diet was prescribed. Under this treatment, the concentration of $2 \mathrm{M} 3 \mathrm{HBA}$ decreased to $27 \mathrm{mmol} / \mathrm{mol}$ creatinine, and $\mathrm{TG}$ lactate, fumarate, and 3-methylglutaconate normalized. Enzymatic activity in fibroblasts showed an intermediate value, $3.81 \mathrm{nmol} / \mathrm{min} / \mathrm{mg}$ protein (controls, $7.27 \pm 1.16$ ), whereas acetoacetyl-CoA thiolase activity was normal. A present, the patient is $10 \mathrm{y}$ old and moderate psychomotor retardation persists.

Patient 2. Patient 2 was a male and was the first-born brother of patient 1 . From the first hours of life, he presented with cyanosis and hypotonia. On the second day of life, cardiomegaly, probably due to congenital cardiopathy, was evident. Biochemical analyses revealed hypoglycemia $(2.2 \mathrm{mM})$, metabolic acidosis, hyperlactatemia $(8 \mathrm{mM})$, and hyperlactaturia. Serum biotinidase, pyruvate carboxylase, and respiratory chain activities in cultured skin fibroblasts were normal. This patient died at 2 mo of age, presumably due to lactic acidosis.

After the diagnosis of his sister, MHBD activity was measured in fibroblasts, which revealed a marked deficiency $(1.5 \mathrm{nmol} / \mathrm{min} / \mathrm{mg}$ protein $)$ whereas acetoacetyl-CoA thiolase activity was normal.

Patient 3. Patient 3 was a male, the first-born child of nonconsanguineous parents. The mother has borderline learning difficulties attributable to a head injury. He presented with dehydration, hypoglycemia, and hypotonia at $2 \mathrm{~h}$ of life. At 3 mo, horizontal nystagmus and absence of reaction to visual stimul were evident. At 7 mo of age, he had marked generalized hypotonia, episodes of hyperextension of the upper limbs, and myoclonus, which were controlled with carbamazepine treatment. At this age, EEG revealed a slow background activity with paroxystic pattern. Magnetic resonance imaging (MRI), performed at 8 mo of age, showed a slight frontotemporal atrophy, and MR spectroscopy demonstrated a high concentration of lactate in basal ganglia, cortex, and white matter. These results suggested a mitochondrial encephalopathy. Amino acids in blood and urine were normal, but blood lactate was consistently high $(7.6-11 \mathrm{mM})$. Urinary organic acid profile showed high excretion of 2M3HBA (89-226 mmol/mol creatinine) and of TG (145-440 $\mathrm{mmol} / \mathrm{mol}$ creatinine). Normal plasma-free carnitine but high esterified carnitine to free carnitine ratio $(0.54$, controls $<0.26)$ was detected. Plasma acylcarnitine profile showed high C5:1 $(1.37 \mu \mathrm{M}$, controls $<0.04)$ and $\mathrm{C} 5-\mathrm{OH}$ species $(0.26 \mu \mathrm{M}$, controls $<0.11)$. MHBD activity in cultured fibroblasts was markedly deficient $(0.8 \mathrm{nmol} / \mathrm{min} / \mathrm{mg}$ protein $)$, whereas acetoacetyl-CoA thiolase activity was normal.

The patient was treated with a vegetarian and isoleucine-restricted diet (45 $\mathrm{mg} / \mathrm{kg} / \mathrm{d})$, carnitine $(50 \mathrm{mg} / \mathrm{kg} / \mathrm{d})$ and carbamazepine. Urinary organic acid excretion decreased to 19 and $10 \mathrm{mmol} / \mathrm{mol}$ creatinine of $2 \mathrm{M} 3 \mathrm{HBA}$ and TG respectively, and plasma acylcarnitine profile normalized, but clinical deteri- oration did not stop, showing optic atrophy, progressive cortical subcortical atrophy and regression in his developmental milestones. He died at 18 mo of age due to a bronchospasm during the course of a catarrh.

Biochemical studies. Organic acids were analyzed by gas chromatographymass spectrometry as TMS derivates. Plasma acylcarnitines were isolated by strong cation-exchange solid phase extraction (11), evaporated to dryness, and esterified with $50 \mu \mathrm{L}$ of butanolic $\mathrm{HCl}(3 \mathrm{~N})$. The butylated dry residue was dissolved in $10 \mu \mathrm{L}$ of matrix $(10 \mathrm{mg} / \mathrm{mL}$ methanolic 2,5 -dihydroxybenzoic acid) and analyzed in a Bruker Reflex III MALDI-TOF mass spectrometer. MHBD in fibroblasts was measured in the reverse direction, as previously described (9)

Molecular studies. Mutation analysis at the cDNA level in the patients was performed by nucleotide sequencing, as previously described (9). Molecular studies were also performed at the genomic level using restriction fragment length polymorphism and $\mathrm{Dde} \mathrm{I}$ and $\mathrm{Bg} / \mathrm{II}$ restriction enzymes to detect $740 \mathrm{~A} \rightarrow \mathrm{G}$ and $388 \mathrm{C} \rightarrow \mathrm{T}$ mutations, respectively. Expression of the mutant cDNA was performed as described in Ofman et al. (9).

\section{RESULTS AND DISCUSSION}

MHBD deficiency is a recently described X-linked inborn error in the metabolism of isoleucine (1). The diagnosis is carried out through the urinary organic acid profile showing elevated excretion of 2M3HBA and TG, without concomitant increase of 2-methylacetoacetate, and is confirmed by measuring MHBD activity in cultured skin fibroblasts (1). So far, only six male patients and one female patient have been described (1-6); all are summarized in Table 1 and compared with the patients reported here.

Our patients 2 and 3 and patient 4, previously reported by Zschocke (1), presented symptoms in the first hours of life. All of them died at an early age, the death of patient 4 was reported by Poll-The et al. (2; personal communication from $\mathrm{J}$. Zschocke). The first symptoms in the remaining patients presented over a range of 9 mo to $6 y$ of age, and all of them are alive with variable degrees of neurologic sequelae.

The most common clinical symptom was speech delay (Table 1); it was observed even in patients with a less severe form of the disease, such as patient 1 . Visual and hearing alterations, hypotonia, and epilepsy are other common symptoms. Brain MRI revealed cerebral atrophy in five out of seven reported patients. Concerning our patients, the MRI was normal in patient 1 in accordance with a less severe form of the disease, whereas patient 3 showed frontotemporal atrophy.

Among the few described patients there is a clear phenotype difference between males and females. The natural history of the disorder in males follows a neurodegenerative course (Table 1), although onset of regression appears to be variable (4). In contrast, the two female patients described to date, patient 1 (this report) and patient 6 (3), do not show regression but do show mild to moderate developmental delay.
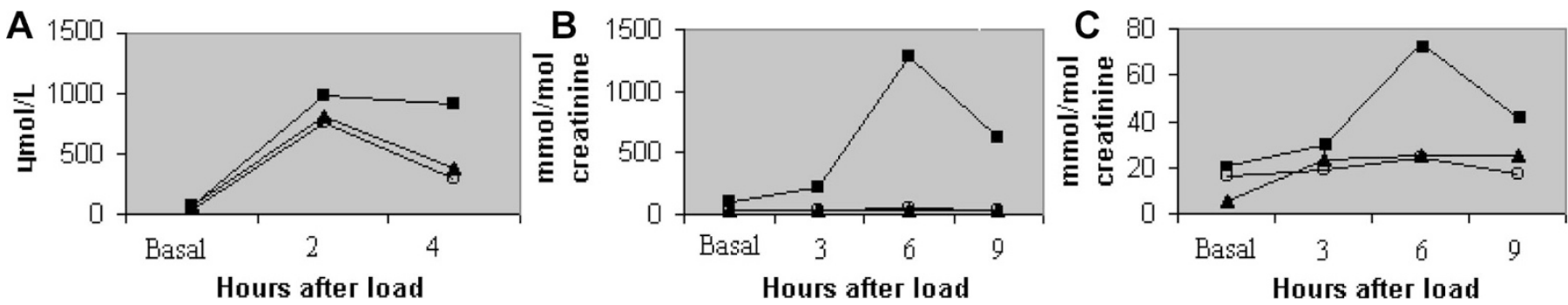

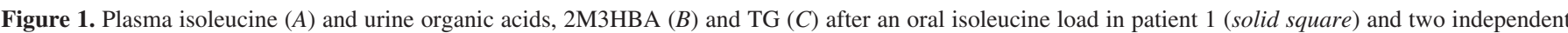
controls (open circle, control 1; solid triangle, control 2). 


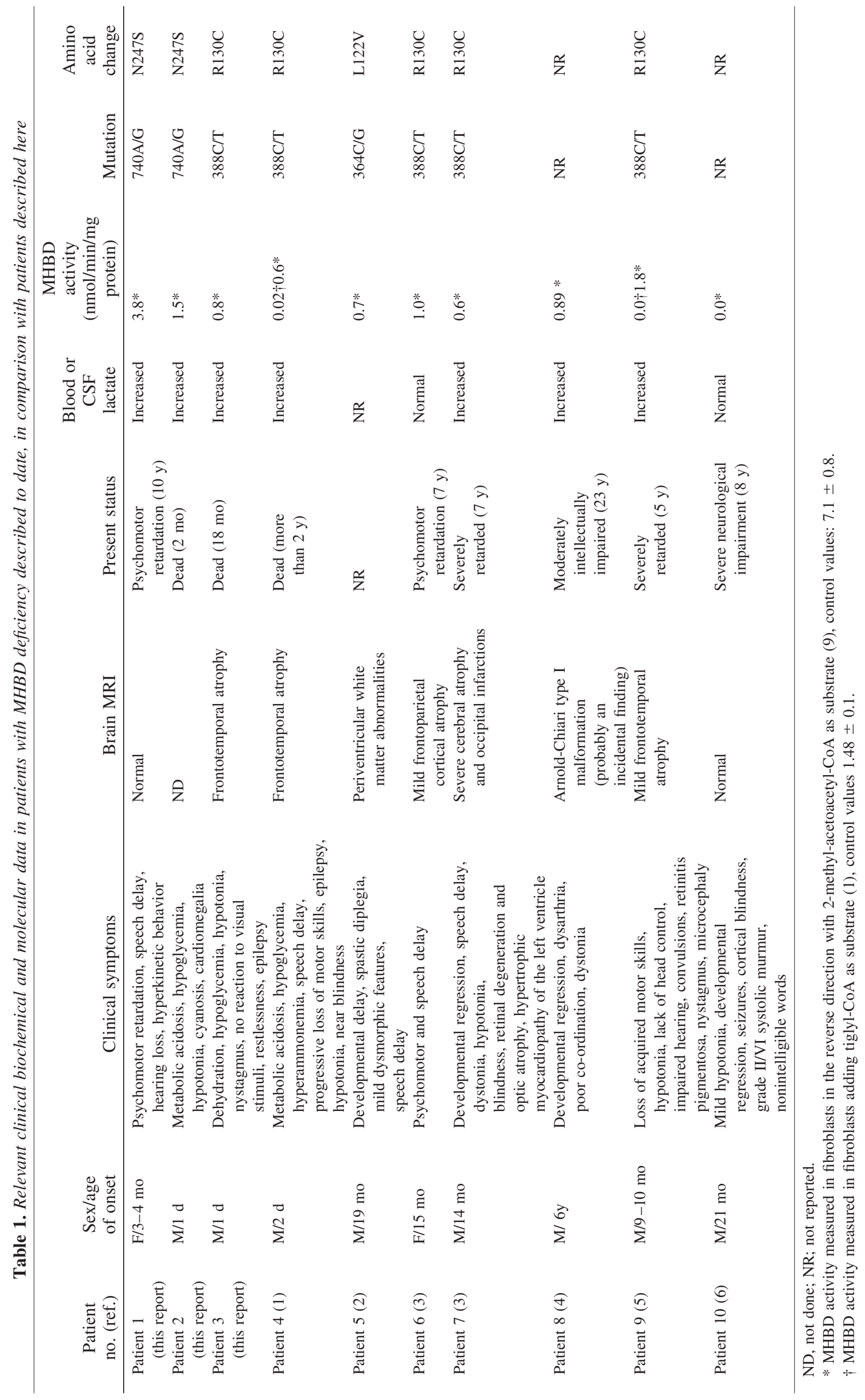


Except for patient 2, from whom no urine sample was available, our patients presented with an increased excretion of 2M3HBA and TG. This excretion was less pronounced in patient 1 , for that reason an oral isoleucine loading test was performed (Fig. 1), showing evidence of a block at the level of MHBD, which was confirmed by enzymatic and molecular studies. Enzymatic studies revealed that patient 1 presented the highest MHBD residual activity described to date, which correlates well with the mild clinical and biochemical phenotype (Table 1), but there are obvious differences in residual enzyme activity depending on the method (see patients 4 and 9 in Table 1 ), which need to be clarified in the future.

Molecular studies in family 1 revealed a new missense mutation in exon $6,740 \mathrm{~A} \rightarrow \mathrm{G}$, which resulted in a replacement of asparagine at position 247 with serine, N247S. It was found in heterozygous form in patient 1 and in her asymptomatic mother, and in hemizygous fashion in her brother (patient 2). Expression studies of the mutant cDNA revealed absence of enzyme activity, confirming the pathogenic nature of the mutation (data not shown). Patient 3 presented a previously described mutation, 388C $\rightarrow \mathrm{T}$ (9), which was also inherited from his mother, who has borderline learning difficulties. This mutation, the commonest among the described patients (Table 1), produced a reduced amount of protein (9).

Our results concerning treatment are somewhat puzzling. The isoleucine restriction did not show any benefit in patient 3 despite normalization of the organic acid profile, but as in other reported patients $(1,3,4)$, the disease remained stable in patient 1 during $1 \mathrm{y}$ of treatment. Therefore, unlike the treatment of other inborn errors of the same metabolic pathway, the use of an isoleucine-restricted diet would not be enough to treat this disease. Lactic acidosis, which is present in almost all patients, might be involved in the etiopathogenesis of the disease and it could be of value to attempt other therapies such us electron acceptors (vitamins) or cofactors (coenzyme Q10) to prevent lactate production (12). However, it remains to be seen whether treatment may improve outcome in presymptomatic patients, as this disease could probably be identified in the neonatal screening programmes by tandem mass spectrometry.

MHBD protein has been described as a multifunctional enzyme, and it has been hypothesized that, in addition to its function in the isoleucine metabolism, MHBD might play an important role in the pathogenesis of Alzheimer's disease (9). This protein appears to have an essential physiologic role in mitochondria, and mutational inactivation of the homologous gene in Drosophila resulted in a lethal phenotype (13). It has recently been demonstrated that human ABAD (also known as ERAB and MHBD) and amyloid- $\beta$ peptide-binding protein directly interact in mitochondria in Alzheimer's disease, and that this interaction inhibits ABAD activity, thus promoting leakage of reactive oxygen species (ROS), mitochondrial dysfunction and cell death (8). The increased concentration of lactate, detected in almost all patients, could be in line with the latter observations or with the secondary complex I deficiency, as has been detected in some patients $(3,4)$. It is noteworthy that, some years ago, a similar organic acid profile was proposed as a metabolic marker for primary complex I deficiency (10); therefore, the differential diagnosis should not only be made with acetoacetyl-CoA thiolase deficiency, but also with mitochondrial respiratory chain deficiencies.

In conclusion, the description of several degrees of clinical severity within a family supports the classical pattern of Xlinked inheritance of this disease. Clinical symptoms as well as the impressive lactic acidosis found in some patients may mimic a mitochondrial disease. The slight clinical and biochemical phenotype in our patient 1 allow us to speculate that the diagnosis of some females might easily be missed. An accurate diagnosis of females is important to prevent the birth of affected offspring. Another consideration to take into account is the possibility of finding adults with neurodegenerative disease brought on by MHBD deficiency.

Acknowledgments. The authors thank Jos. P.N. Ruiter for enzyme measurements, and Conxita Llordés and Carlota Ogg for their excellent technical assistance.

\section{REFERENCES}

1. Zschocke J, Ruiter JP, Brand J, Lindner M, Hoffmann GF, Wanders RJ, Mayatepek E 2000 Progressive infantile neurodegeneration caused by 2-methyl-3-hydroxybutyryl-CoA dehydrogenase deficiency: a novel inborn error of branched-chain fatty acid and isoleucine metabolism. Pediatr Res 48:852-855

2. Poll-The BT, Wanders RJ, Ruiter JPN, Ofman R, Majoie CB, Barth PG, Duran M 2004 Spastic diplegia and periventricular white matter abnormalities in 2-methyl-3hydroxybutyryl-CoA dehydrogenase deficiency, a defect of isoleucine metabolism: differential diagnosis with hypoxic-ischemic brain diseases. Mol Genet Metab 81:295-299

3. Ensenauer R, Niederhoff H, Ruiter JP, Wanders RJ, Schwab KO, Brandis M, Lehnert W 2002 Clinical variability in 3-hydroxy-2-methylbutyryl-CoA dehydrogenase deficiency. Ann Neurol 51:656-659

4. Olpin SE, Pollitt RJ, McMenamin J, Manning NJ, Besley G, Ruiter JP, Wanders RJ 2002 2-Methyl-3-hydroxybutyryl-CoA dehydrogenase deficiency in a 23-year-old man. J Inherit Metab Dis 25:477-482

5. Sass JO, Forstner R, Sperl W 2004 2-Methyl-3-hydroxybutyryl-CoA dehydrogenase deficiency: impaired catabolism of isoleucine presenting as neurodegenerative disease. Brain Dev 26:12-14

6. Sutton VR, O'Brien WE, Clark GD, Kim J, Wanders RJ 2003 3-hydroxy-2methylbutyryl-CoA dehydrogenase deficiency. J Inherit Metab Dis 26:69-71

7. Yan SD, Fu J, Soto C, Chen X, Zhu H, Al-Mohanna F, Collison K, Zhu A, Stern E, Saido T, Tohyama M, Ogawa S, Roher A, Stern D 1997 An intracellular protein that binds amyloid- $\beta$ peptide and mediates neurotoxicity in Alzheimer's disease. Nature 389:689-695

8. Lustbader JW, Cirilli M, Lin C, Xu HW, Takuma K, Wang N, Caspersen C, Chen X, Pollak S, Chaney M, Trinchese F, Liu S, Gunn-Moore F, Lue LF, Walker DG, Kuppusamy P, Zewier ZL, Arancio O, Stern D, Yan SS, Wu H 2004 ABAD directly links $\mathrm{A} \beta$ to mitochondrial toxicity in Alzheimer's disease. Science 304:448-452

9. Ofman R, Ruiter JP, Feenstra M, Duran M, Poll-The BT, Zschocke J, Ensenauer R, Lehnert W, Sass JO, Sperl W, Wanders RJ 2003 2-Methyl-3-hydroxybutyryl-CoA dehydrogenase deficiency is caused by mutations in the HADH2 gene. Am J Hum Genet 72:1300-1307

10. Bennett MJ, Sherwood WG, Gibson KM, Burlina AB 1993 Secondary inhibition of multiple NAD-requiring dehydrogenases in respiratory chain complex I deficiency: possible metabolic markers for the primary defect. J Inherit Metab Dis 16:560-562

11. Costa CG, Struys EA, Bootsma A, ten Brink HJ, Dorland L, Tavares de Almeida I, Duran M, Jakobs C 1997 Quantitative analysis of plasma acylcarnitines using gas chromatography chemical ionization mass fragmentography. J Lipid Res 38:173-182

12. Marriage BJ, Clandinin MT, Mac Donald IM, Glerum DM 2004 Cofactor treatment improves ATP synthetic capacity in patients with oxidative phosphorylation disorders. Mol Genet Metab 81:263-272

13. Torroja L, Ortuño-Sahagún D, Ferrús A, Hämmerle B, Barbas JA 1998 Scully, an essential gene of Drosophila, is homologous to mammalian mitochondrial type II L-3-hydroxyacyl-CoA dehydrogenase/amyloid- $\beta$ peptide-binding protein. J Cell Biol 141:1009-1017 